\title{
CONNECTIVITY AND DENSITY IN THE SET OF FRAMELETS
}

\author{
MARCIN BOWNIK
}

Abstract. We show that the set of all framelets is pathwise connected and dense both in $L^{2}(\mathbb{R}, d x)$ and $L^{2}(\mathbb{R}, d \xi /|\xi|)$ norms. This answers an open problem posed by Larson $[12,13]$.

\section{Introduction}

One of the fundamental areas of the theory of wavelets is the investigation of properties of the collection of all wavelets as a subset of $L^{2}(\mathbb{R})$. Probably the most prominent problem in this area is a question whether the collection of all orthonormal wavelets (as a subset of the unit sphere) is connected in $L^{2}(\mathbb{R})$ norm $[5,17]$. More generally, one can also ask whether the collection of all tight frame wavelets (as a subset of the unit ball) is connected in $L^{2}(\mathbb{R})$ norm [7].

While these two questions remain open so far, there are several strong results indicating that the answer to both questions might be positive. The joint work of the Wutam consortium showed that the class of all multiresolution analysis (MRA) wavelets is connected [17]. Speegle showed that the class of all minimally supported frequency (MSF) wavelets is also connected. On the other front, Paluszyński, Šikić, Weiss, and Xiao showed the connectivity for the class of MRA tight frame wavelets $[14,15]$. Finally, Garrigós, Hernández, Šikić, Soria, Weiss, and Wilson showed that the class of all tight framelets satisfying very mild conditions on their spectrum is also connected $[7,8]$.

Despite this, little attention has been given so far to the study of the properties of the collection of all (not necessarily tight) frame wavelets. The goal of this work is to change this by showing that the set of all frame wavelets is connected. A remarkable feature of our techniques is that the connectivity is shown not only in the usual $L^{2}(\mathbb{R})$ norm, but also in "multiplicative" $L^{2}(\mathbb{R}, d \xi /|\xi|)$ norm. Furthermore, we prove that the collection of all wavelet frames is also dense in both of these norms.

We start by recalling some basic terminology. A frame wavelet, or in short a framelet, is a function $\psi \in L^{2}(\mathbb{R})$ such that the affine system

$$
\psi_{j, k}(x)=2^{j / 2} \psi\left(2^{j} x-k\right), \quad j, k \in \mathbb{Z},
$$

forms a frame for $L^{2}(\mathbb{R})$. Hence, we require the existence of constants $0<c_{0} \leq c_{1}<\infty$ such that

$$
c_{0}\|f\|^{2} \leq \sum_{j, k \in \mathbb{Z}}\left|\left\langle f, \psi_{j, k}\right\rangle\right|^{2} \leq c_{1}\|f\|^{2} \quad \text { for all } f \in L^{2}(\mathbb{R}) .
$$

Received by the editors September 16, 2005.

2000 Mathematics Subject Classification. Primary: 42C40.

The author was partially supported by the NSF grant DMS-0441817. 
We say that the affine system is Bessel if only the upper bound holds in (1.1). Let $\mathcal{W}$ be the set of all framelets

$$
\mathcal{W}=\left\{\psi \in L^{2}(\mathbb{R}): \psi \text { is a framelet }\right\} .
$$

It is well-known that $\mathcal{W} \subset L^{2}(\mathbb{R})$ is also a subset of the space $L^{2}(\mathbb{R}, d \xi /|\xi|)$, which consists of all functions $f$ such that

$$
\|f\|_{L^{2}(\mathbb{R}, d \xi /|\xi|)}=\left(\int_{\mathbb{R}}|\hat{f}(\xi)|^{2} \frac{d \xi}{|\xi|}\right)^{1 / 2}<\infty
$$

where the Fourier transform of $f$ is defined as

$$
\hat{f}(\xi)=\int_{\mathbb{R}} f(x) e^{-2 \pi i x \xi} d x .
$$

This is a consequence of the fact that

$$
c_{0} \leq \sum_{j \in \mathbb{Z}}\left|\hat{\psi}\left(2^{j} \xi\right)\right|^{2} \leq c_{1} \quad \text { for a.e. } \xi \in \mathbb{R} .
$$

Hence, $\mathcal{W}$ is a subset of the intersection $L^{2}(\mathbb{R}) \cap L^{2}(\mathbb{R}, d \xi /|\xi|)$, which can be identified with the space

$$
L_{*}^{2}(\mathbb{R}):=L^{2}(\mathbb{R},(1+1 /|\xi|) d \xi)=\left\{f:|| f \|_{*}=\left(\int_{\mathbb{R}}|\hat{f}(\xi)|^{2}(1+1 /|\xi|) d \xi\right)^{1 / 2}<\infty\right\} .
$$

The space $L_{*}^{2}(\mathbb{R})$ appears to be a natural choice for the study of the set of wavelets and it was already introduced by Garrigós and Speegle [9]. There, the authors proved that the set of all orthonormal wavelets and also the set of all tight framelets are both complete in $L_{*}^{2}(\mathbb{R})$ norms. However, none of these sets is complete in $L^{2}(\mathbb{R})$ norm nor in $L^{2}(\mathbb{R}, d \xi /|\xi|)$ norm alone, see $[9]$.

\section{Density}

The question whether the set of all framelets is dense in $L^{2}(\mathbb{R})$ was posed by Larson $[12,13]$ in 1996. In this section we give a positive answer to his question. We need the following standard result, see [11, Theorem 13.0.1].

Lemma 2.1. Suppose that $f \in L^{2}(\mathbb{R})$ is such that $\hat{f} \in L^{\infty}(\mathbb{R})$ and

$$
\begin{aligned}
& \hat{f}(\xi)=O\left(|\xi|^{\delta}\right) \quad \text { as } \xi \rightarrow 0, \\
& \hat{f}(\xi)=O\left(|\xi|^{-1 / 2-\delta}\right) \quad \text { as }|\xi| \rightarrow \infty,
\end{aligned}
$$

for some $\delta>0$. Then the affine system $\left\{D^{j} T_{k} f: j, k \in \mathbb{Z}\right\}$ is a Bessel sequence, where $D f(x)=\sqrt{2} f(2 x), T_{k} f(x)=f(x-k)$.

Theorem 2.1. The collection $\mathcal{W}$ of all framelets is dense in $L_{*}^{2}(\mathbb{R})$.

Proof. Let

$$
\mathcal{D}=\left\{f \in L^{2}(\mathbb{R}): \hat{f} \in L^{\infty}(\mathbb{R}) \text { and } \exists R>0 \text { supp } \hat{f} \subset[-R,-1 / R] \cup[1 / R, R]\right\} .
$$

Since $\mathcal{D}$ is a dense subspace of $L_{*}^{2}(\mathbb{R})$, it suffices to show that

$$
\forall f \in \mathcal{D} \exists \text { a sequence of framelets }\left\{\psi_{j}\right\}_{j \in \mathbb{N}} \text { such that } \lim _{j \rightarrow \infty}\left\|f-\psi_{j}\right\|_{*}=0 \text {. }
$$


Take any $f \in \mathcal{D}$. Given $\delta>0$ and $r>1$ define a function $\psi=\psi(f, r, \delta) \in L_{*}^{2}(\mathbb{R})$ by

$$
\begin{array}{r}
\psi=\psi^{0}+\psi^{1}, \quad \text { where } \hat{\psi}^{0}=\delta \mathbf{1}_{S_{r}}, \quad \hat{\psi}^{1}=\hat{f} \mathbf{1}_{Z_{r}}, \\
S_{r}=\left[-2^{-r},-2^{-r-1}\right] \cup\left[2^{-r-1}, 2^{-r}\right], \quad Z_{r}=\mathbb{R} \backslash \bigcup_{k \in \mathbb{Z}}\left(k+S_{r}\right) .
\end{array}
$$

Since supp $\hat{\psi}^{0} \subset[-1 / 2,1 / 2]$, it is clear from the characterizing equations [10, Section 7.1] that $\psi^{0}$ is a tight framelet (with the frame constant $\delta^{2}$ ). On the other hand, while $\psi^{1}$ does not have to be a framelet itself, the affine system generated by it must be Bessel by Lemma 2.1 .

First, we claim that $\psi$ given by (2.2) is a framelet. By the above observation and a basic perturbation argument, the affine system generated by $\psi$ is Bessel as well. Hence, it suffices to show that the lower frame bound of $\psi$ is positive; in fact, at least $\delta^{2}$. More precisely, it is enough to show that for every $j \in \mathbb{Z}$,

$$
\sum_{k \in \mathbb{Z}}\left|\left\langle f, \psi_{j, k}\right\rangle\right|^{2} \geq \delta^{2} \int_{2^{j} S_{r}}|\hat{f}(\xi)|^{2} d \xi
$$

Indeed, (2.3) implies that

$$
\sum_{j \in \mathbb{Z}} \sum_{k \in \mathbb{Z}}\left|\left\langle f, \psi_{j, k}\right\rangle\right|^{2} \geq \delta^{2} \sum_{j \in \mathbb{Z}} \int_{2^{j} S_{r}}|\hat{f}(\xi)|^{2} d \xi=\delta^{2}\|f\|^{2}
$$

since the family $\left\{2^{j} S_{r}\right\}_{j \in \mathbb{Z}}$ partitions $\mathbb{R}$ modulo null sets. Finally, to prove (2.3) we use a standard periodization argument

$$
\begin{aligned}
& \sum_{k \in \mathbb{Z}}\left|\left\langle f, \psi_{j, k}\right\rangle\right|^{2}=\sum_{k \in \mathbb{Z}}\left|\left\langle f, D^{j} T_{k} \psi\right\rangle\right|^{2}=\sum_{k \in \mathbb{Z}}\left|\left\langle D^{-j} f, T_{k} \psi\right\rangle\right|^{2} \\
& =\int_{-1 / 2}^{1 / 2}\left|\sum_{l \in \mathbb{Z}} \widehat{D^{-j} f}(\xi+l) \overline{\hat{\psi}(\xi+l)}\right|^{2} d \xi \geq 2^{j} \int_{S_{r}}\left|\sum_{l \in \mathbb{Z}} \hat{f}\left(2^{j}(\xi+l)\right) \overline{\hat{\psi}(\xi+l)}\right|^{2} d \xi \\
& =2^{j} \int_{S_{r}}\left|\hat{f}\left(2^{j} \xi\right) \hat{\psi}^{0}(\xi)\right|^{2} d \xi=\delta^{2} \int_{2^{j} S_{r}}|\hat{f}(\xi)|^{2} d \xi
\end{aligned}
$$

where in the penultimate step we have used that $\operatorname{supp} \hat{\psi}^{0}=S_{r} \subset[-1 / 2,1 / 2]$ and $\operatorname{supp} \hat{\psi}^{1} \subset Z_{r}$ lies outside the periodization of $S_{r}$.

Next, we estimate the distance between $\psi$ and $f$ in $L_{*}^{2}(\mathbb{R})$ norm

$$
\begin{aligned}
\|f-\psi\|_{*}^{2} & =\int_{\mathbb{R}}|\hat{f}(\xi)-\hat{\psi}(\xi)|^{2}(1+1 /|\xi|) d \xi \\
& =\int_{S_{r}}|\hat{f}(\xi)-\delta|^{2}(1+1 /|\xi|) d \xi+\int_{\mathbb{R} \backslash S_{r}}\left|\hat{f}(\xi)-\hat{\psi}^{1}(\xi)\right|^{2}(1+1 /|\xi|) d \xi
\end{aligned}
$$


A simple calculation shows that the first integral equals $\delta^{2}\left(2 \ln (2)+2^{-r}\right)$ for sufficiently large $r$, i.e., for $r>\ln (R) / \ln (2)$. Since $\hat{\psi}^{1}=\mathbf{1}_{Z_{r}} \hat{f}$, the second integral equals

$$
\begin{aligned}
\int_{\bigcup_{k \in \mathbb{Z} \backslash\{0\}} k+S_{r}}|\hat{f}(\xi)|^{2}(1+1 /|\xi|) d \xi & =\sum_{k \in \mathbb{Z}, 0 \neq|k| \leq R+1} \int_{k+S_{r}}|\hat{f}(\xi)|^{2}(1+1 /|\xi|) d \xi \\
& \leq 2(R+1)|| \hat{f} \|_{\infty}^{2}\left(\left|S_{r}\right|+\int_{\mathbb{R}} \mathbf{1}_{1+S_{r}} d \xi /|\xi|\right) \\
& \leq 2(R+1)|| \hat{f} \|_{\infty}^{2}\left(2^{-r}+\ln \left(\left(1+2^{-r}\right) /\left(1-2^{-r}\right)\right)\right) .
\end{aligned}
$$

Hence,

$$
\|f-\psi(f, r, \delta)\|_{*}^{2} \rightarrow 0 \quad \text { as } \delta \rightarrow 0 \text { and } r \rightarrow \infty .
$$

Therefore, functions $\psi=\psi(f, r, \delta)$ are the required framelets approximating $f$ in $L_{*}^{2}(\mathbb{R})$ norm.

Remark 2.1. A closer inspection of the proof of Theorem 2.1 shows that the set

$$
\mathcal{W}_{d}=\left\{\psi \in L^{2}(\mathbb{R}): \psi \text { is a framelet which has a dual framelet }\right\} .
$$

is also dense in $L_{*}^{2}(\mathbb{R})$ norm. Recall that $\phi$ is a dual framelet to a framelet $\psi$ if

$$
f=\sum_{j, k \in \mathbb{Z}}\left\langle f, \psi_{j, k}\right\rangle \phi_{j, k} \quad \text { for all } f \in L^{2}(\mathbb{R}) .
$$

It is well known, see [6], that $(\phi, \psi)$ is a pair of dual framelets if and only if $\left\{\phi_{j, k}\right.$ : $j, k \in \mathbb{Z}\}$ and $\left\{\psi_{j, k}: j, k \in \mathbb{Z}\right\}$ are Bessel sequences and the following characterizing equations hold

$$
\begin{aligned}
\sum_{j \in \mathbb{Z}} \hat{\phi}\left(2^{j} \xi\right) \overline{\hat{\psi}\left(2^{j} \xi\right)} & =1 \quad \text { for a.e. } \xi \\
\sum_{j=0}^{\infty} \hat{\phi}\left(2^{j} \xi\right) \overline{\hat{\psi}\left(2^{j}(\xi+q)\right)}=0 & \text { for a.e. } \xi \text { and for odd } q \in \mathbb{Z} .
\end{aligned}
$$

It is also well known that $\mathcal{W}_{d}$ is a proper subset of $\mathcal{W}$, see e.g. [3, Theorem 2].

Indeed, to verify the density of $\mathcal{W}_{d}$ it suffices to show that $\psi=\psi(f, r, \delta)$ given by (2.2) has a dual framelet. Let $\phi \in L^{2}(\mathbb{R})$ be given by

$$
\hat{\phi}=\delta^{-1} \mathbf{1}_{S_{r}}, \quad \text { where } S_{r}=\left[-2^{-r},-2^{-r-1}\right] \cup\left[2^{-r-1}, 2^{-r}\right] .
$$

Since $r>1$, it is easy to see that $\phi$ is a tight framelet. Moreover, (2.4) is immediate. To prove (2.5) it suffices to observe that if $\hat{\phi}\left(2^{j} \xi\right) \neq 0$ then $2^{j} \xi \in S_{r}$. Consequently, $2^{j} \xi+q \notin \operatorname{supp} \hat{\psi}$ for any $q \in \mathbb{Z} \backslash\{0\}$ by the choice of the set $Z_{r}$ and (2.2). Hence, $\hat{\psi}\left(2^{j}(\xi+q)\right)=0$ and $(2.5)$ must hold. This shows that $(\phi, \psi)$ is a pair of dual framelets and completes the proof of Remark 2.1.

\section{Connectivity}

In this section we show the connectivity of the set of frame wavelets.

Theorem 3.1. The collection $\mathcal{W}$ of all framelets is pathwise connected in $L_{*}^{2}(\mathbb{R})$. 
Proof. Let $\psi^{0} \in \mathcal{W}$ be an arbitrary framelet. Let $\psi^{1}$ be a fixed tight framelet defined by

$$
\hat{\psi}^{1}=\mathbf{1}_{[-1 / 2,-1 / 4] \cup[1 / 4,1 / 2]} .
$$

We will prove that there exists a path of framelets $\left\{\psi^{t}\right\}_{t \in[0,1]} \subset \mathcal{W}$ such that that map $t \mapsto \psi^{t}$ is continuous in $L_{*}^{2}(\mathbb{R})$ norm. In fact, this path is given explicitly by

$$
\begin{aligned}
& \psi^{t}=\phi^{0}+\phi^{1}, \quad \text { where } \hat{\phi}^{0}=\hat{\psi}^{0} \mathbf{1}_{Z_{t}}, \quad \hat{\phi}^{1}=t \mathbf{1}_{S_{t}}, \quad t \in[0,1], \\
& S_{t}=[-t / 2,-t / 4] \cup[t / 4, t / 2], \quad Z_{t}=\mathbb{R} \backslash \bigcup_{k \in \mathbb{Z}}(k+[-t / 2, t / 2]) .
\end{aligned}
$$

First, we claim that $\psi^{t} \in \mathcal{W}$ for every $t \in(0,1)$. As in the proof of Theorem 2.1 we use a standard periodization argument. For any $f \in L^{2}(\mathbb{R})$ and $j \in \mathbb{Z}$,

$$
\begin{aligned}
\sum_{k \in \mathbb{Z}}\left|\left\langle f,\left(\psi^{t}\right)_{j, k}\right\rangle\right|^{2}= & \int_{-1 / 2}^{1 / 2}\left|\sum_{l \in \mathbb{Z}} \widehat{D^{-j} f}(\xi+l) \overline{\hat{\psi}^{t}(\xi+l)}\right|^{2} d \xi \\
= & 2^{j} \int_{-t / 2}^{t / 2}\left|\sum_{l \in \mathbb{Z}} \hat{f}\left(2^{j}(\xi+l)\right) \overline{\hat{\psi}^{t}(\xi+l)}\right|^{2} d \xi \\
& \quad+2^{j} \int_{[-1 / 2,1 / 2] \backslash[-t / 2, t / 2]} \mid \sum_{l \in \mathbb{Z}} \hat{f}\left(\left.2^{j}(\xi+l) \overline{\hat{\psi}^{t}(\xi+l)}\right|^{2} d \xi\right. \\
= & 2^{j} \int_{-t / 2}^{t / 2}\left|\sum_{l \in \mathbb{Z}} \hat{f}\left(2^{j}(\xi+l)\right) \overline{\hat{\phi}^{1}(\xi+l)}\right|^{2} d \xi \\
& +2^{j} \int_{[-1 / 2,1 / 2] \backslash[-t / 2, t / 2]}\left|\sum_{l \in \mathbb{Z}} \hat{f}\left(2^{j}(\xi+l)\right) \overline{\hat{\phi}^{0}(\xi+l)}\right|^{2} d \xi
\end{aligned}
$$

The last two integrals can be easily computed

$$
\begin{aligned}
2^{j} \int_{-t / 2}^{t / 2} \mid & \left.\sum_{l \in \mathbb{Z}} \hat{f}\left(2^{j}(\xi+l)\right) \overline{\hat{\phi}^{1}(\xi+l)}\right|^{2} d \xi=2^{j} t^{2} \int_{S_{t}}\left|\hat{f}\left(2^{j} \xi\right)\right|^{2} d \xi=t^{2} \int_{2^{j} S_{t}}|\hat{f}(\xi)|^{2} d \xi . \\
& 2^{j} \int_{[-1 / 2,1 / 2] \backslash[-t / 2, t / 2]}\left|\sum_{l \in \mathbb{Z}} \hat{f}\left(2^{j}(\xi+l)\right) \overline{\hat{\phi}^{0}(\xi+l)}\right|^{2} d \xi \\
\leq & 2^{j} \int_{-1 / 2}^{1 / 2}\left|\sum_{l \in \mathbb{Z}} \hat{f}\left(2^{j}(\xi+l)\right) \overline{\hat{\psi}^{0}(\xi+l)}\right|^{2} d \xi=\sum_{k \in \mathbb{Z}}\left|\left\langle f,\left(\psi^{0}\right)_{j, k}\right\rangle\right|^{2} .
\end{aligned}
$$

Summing the above formulas over $j \in \mathbb{Z}$ we have

$$
t^{2}\|f\|^{2} \leq \sum_{j \in \mathbb{Z}} \sum_{k \in \mathbb{Z}}\left|\left\langle f,\left(\psi^{t}\right)_{j, k}\right\rangle\right|^{2} \leq t^{2}\|f\|^{2}+\sum_{j \in \mathbb{Z}} \sum_{k \in \mathbb{Z}}\left|\left\langle f,\left(\psi^{0}\right)_{j, k}\right\rangle\right|^{2} .
$$

This proves that $\psi^{t} \in \mathcal{W}$ for each $t \in[0,1]$. 
Finally, the continuity of the path $\left\{\psi^{t}\right\}_{t \in[0,1]}$ in $L_{*}^{2}(\mathbb{R})$ is a consequence of the Lebesgue Dominated Convergence Theorem. Indeed, for any $0 \leq t<t^{\prime} \leq 1$ we have

$$
\begin{aligned}
\left\|\psi^{t}-\psi^{t^{\prime}}\right\|_{*} \leq & \left(\int_{\mathbb{R}}\left|\hat{\psi}^{0}(\xi)\right|^{2} \mathbf{1}_{Z_{t} \backslash Z_{t^{\prime}}}(1+1 /|\xi|) d \xi\right)^{1 / 2} \\
& +\left(\int_{\mathbb{R}}\left|t \mathbf{1}_{S_{t}}-t^{\prime} \mathbf{1}_{S_{t^{\prime}}}\right|^{2}(1+1 /|\xi|) d \xi\right)^{1 / 2} .
\end{aligned}
$$

A simple application of the Lebesgue Dominated Convergence Theorem shows that for each $t \in[0,1],\left\|\psi^{t}-\psi^{t^{\prime}}\right\|_{*} \rightarrow 0$ as $t^{\prime} \rightarrow t$, which completes the proof of Theorem 3.1 .

Remark 3.1. The arguments used in the proof of Theorem 3.1 show that the set of dual framelets $\mathcal{W}_{d}$ is also pathwise connected in $L_{*}^{2}(\mathbb{R})$ norm. Indeed, it suffices to observe that each $\psi^{t}, t \in(0,1]$, given by $(3.1)$ is in $\mathcal{W}_{d}$, regardless whether $\psi^{0} \in \mathcal{W}_{d}$, or not. In particular, if $\psi^{0} \in \mathcal{W}_{d}$, then we would have a continuous path $\left\{\psi^{t}\right\}_{t \in[0,1]}$ inside $\mathcal{W}_{d}$.

To prove Remark 3.1, let $\phi^{t} \in L^{2}(\mathbb{R})$ be given by $\hat{\phi}^{t}=t^{-1} \mathbf{1}_{S_{t}}$, where $S_{t}=$ $[-t / 2,-t / 4] \cup[t / 4, t / 2]$ and $0<t \leq 1$. As in the proof of Remark 2.1, it is not hard to verify that $\left(\phi^{t}, \psi^{t}\right)$ is a pair of dual framelets employing the characterizing equations (2.4) and (2.5).

\section{Extensions to higher dimensions}

Theorems 2.1 and 3.1 are also valid for more general framelets associated with expansive dilations in $\mathbb{R}^{n}$. Recall that $n \times n$ real matrix $A$ is said to be expansive if all eigenvalues $\lambda$ of $A$ satisfy $|\lambda|>1$. A quasi-norm associated with expansive matrix $B$ is a measurable mapping $\rho: \mathbb{R}^{n} \rightarrow[0, \infty)$, such that

(i) $\rho(\xi)=0 \Longleftrightarrow \xi=0$,

(ii) $\rho(B \xi)=|\operatorname{det} B| \rho(\xi)$ for all $\xi \in \mathbb{R}^{n}$,

(iii) $\exists c>0 \rho\left(\xi+\xi^{\prime}\right) \leq c\left(\rho(\xi)+\rho\left(\xi^{\prime}\right)\right)$ for all $\xi, \xi^{\prime} \in \mathbb{R}^{n}$.

Basic properties about quasi-norms, including the fact that all quasi-norms associated with a fixed dilation are equivalent, can be found in [2].

In this setting, a framelet is a function $\psi \in L^{2}\left(\mathbb{R}^{n}\right)$, such that the system

$$
\psi_{j, k}(x)=|\operatorname{det} A|^{j / 2} \psi\left(A^{j} x-k\right), \quad j \in \mathbb{Z}, k \in \mathbb{Z}^{n},
$$

forms a frame for $L^{2}\left(\mathbb{R}^{n}\right)$. As before, let $\mathcal{W}=\mathcal{W}_{A}$ be the set of all framelets

$$
\mathcal{W}_{A}=\left\{\psi \in L^{2}\left(\mathbb{R}^{n}\right): \psi \text { is a framelet }\right\} .
$$

It is well-known that every $\psi \in \mathcal{W}_{A}$ with frame constants $0<c_{0} \leq c_{1}<\infty$ satisfies the inequality

$$
c_{0} \leq \sum_{j \in \mathbb{Z}}\left|\hat{\psi}\left(B^{j} \xi\right)\right|^{2} \leq c_{1} \quad \text { for a.e. } \xi \in \mathbb{R}^{n},
$$

where $B=A^{*}$. Therefore, $\mathcal{W}_{A} \subset L^{2}\left(\mathbb{R}^{n}\right)$ is also a subset of the space $L^{2}\left(\mathbb{R}^{n}, d \xi / \rho(\xi)\right)$, which consists of all functions $f$ such that

$$
\|f\|_{L^{2}\left(\mathbb{R}^{n}, d \xi / \rho(\xi)\right)}=\left(\int_{\mathbb{R}^{n}}|\hat{f}(\xi)|^{2} \frac{d \xi}{\rho(\xi)}\right)^{1 / 2}<\infty,
$$


where $\rho$ is a quasi-norm associated with the dilation $B=A^{*}$. Indeed, this is a consequence of (4.1) and a calculation as in [1, Theorem 2.5]. Hence, $\mathcal{W}$ is a subset of the intersection $L^{2}\left(\mathbb{R}^{n}\right) \cap L^{2}(\mathbb{R}, d \xi / \rho(\xi))$, which can be identified with the space

$$
L_{\rho}^{2}\left(\mathbb{R}^{n}\right):=L^{2}(\mathbb{R},(1+1 / \rho(\xi)) d \xi) .
$$

Then we can generalize Theorem 2.1 and 3.1.

Theorem 4.1. The collection $\mathcal{W}_{A}$ of all framelets associated with an expansive dilation $A$ is dense and pathwise connected in $L_{\rho}^{2}\left(\mathbb{R}^{n}\right)$.

Proof. To show density of $\mathcal{W}_{A}$ we mimic the proof of Theorem 2.1. Let

$$
\mathcal{D}=\left\{f \in L^{2}\left(\mathbb{R}^{n}\right): \hat{f} \in L^{\infty}\left(\mathbb{R}^{n}\right) \text { and } \exists R>0 \operatorname{supp} \hat{f} \subset B(0, R) \backslash B(0,1 / R)\right\} .
$$

be a dense subspace of $L_{\rho}^{2}\left(\mathbb{R}^{n}\right)$. Since $B=A^{*}$ is expansive, we can find an ellipsoid $E$ satisfying $\lambda E \subset B(E)$ for some $\lambda>1$, see [2]. Moreover, by rescaling we can also assume that $E \subset[-1 / 2,1 / 2]^{n}$.

Given $f \in \mathcal{D}, \delta>0$ and $r \in \mathbb{N}$ define a function $\psi=\psi(f, r, \delta) \in L_{\rho}^{2}\left(\mathbb{R}^{n}\right)$ by

$$
\begin{gathered}
\psi=\psi^{0}+\psi^{1}, \quad \text { where } \hat{\psi}^{0}=\delta \mathbf{1}_{S_{r}}, \quad \hat{\psi}^{1}=\hat{f} \mathbf{1}_{Z_{r}}, \\
S_{r}=B^{-r} E \backslash B^{-r-1} E, \quad Z_{r}=\mathbb{R}^{n} \backslash \bigcup_{k \in \mathbb{Z}^{n}}\left(k+S_{r}\right) .
\end{gathered}
$$

A similar argument as in Theorem 2.1 shows that $\psi=\psi(f, r, \delta)$ is a framelet and

$$
\|f-\psi(f, r, \delta)\|_{\rho} \rightarrow 0 \quad \text { as } \delta \rightarrow 0 \text { and } r \rightarrow \infty .
$$

Likewise, to show connectivity of $\mathcal{W}_{A}$ it suffices to find a continuous path of framelets $\left\{\psi^{t}\right\}_{t \in[0,1]} \subset \mathcal{W}_{A}$, where $\psi^{0} \in \mathcal{W}_{A}$ is an arbitrary framelet and $\psi^{1} \in \mathcal{W}_{A}$ is a fixed tight framelet defined by

$$
\hat{\psi}^{1}=\mathbf{1}_{E \backslash B^{-1}(E)} .
$$

Following the approach of Theorem 3.1, we can define this path explicitly by

$$
\begin{aligned}
& \psi^{t}=\phi^{0}+\phi^{1}, \quad \text { where } \hat{\phi}^{0}=\hat{\psi}^{0} \mathbf{1}_{Z_{t}}, \quad \hat{\phi}^{1}=t \mathbf{1}_{S_{t}}, \quad t \in[0,1], \\
& S_{t}=t\left(E \backslash B^{-1}(E)\right), \quad Z_{t}=\mathbb{R} \backslash \bigcup_{k \in \mathbb{Z}^{n}}(k+t E) .
\end{aligned}
$$

An easy adaptation of the proof of Theorem 3.1 shows that $\psi^{t} \in \mathcal{W}_{A}$ for all $t \in[0,1]$ and $t \mapsto \psi^{t}$ is continuous in $L_{\rho}^{2}\left(\mathbb{R}^{n}\right)$ norm.

Remark 4.1. As an immediate consequence of Theorem 4.1 we conclude that the set of all framelets $\mathcal{W}$ (or its higher dimensional counterpart $\mathcal{W}_{A}$ ) is not complete in $L_{*}^{2}(\mathbb{R})$ norm (or $L_{\rho}^{2}\left(\mathbb{R}^{n}\right)$ norm). This is in stark contrast with the result of Garrigós and Speegle [9], which established the completness of the set of tight framelets.

This suggest that it might be worthwhile to consider a more restricted class of framelets satisfying some prescribed frame bounds $0<c_{0} \leq c_{1}<\infty$. More precisely, let

$$
\mathcal{W}\left(c_{0}, c_{1}\right)=\left\{\psi \in L^{2}(\mathbb{R}): \psi \text { satisfies }(1.1)\right\} .
$$

While it is clear that the density of $\mathcal{W}\left(c_{0}, c_{1}\right)$ fails, see the example below, it is an open problem whether the set $\mathcal{W}\left(c_{0}, c_{1}\right)$ is connected in $L_{*}^{2}(\mathbb{R})$ (or even in $L^{2}(\mathbb{R})$ ) norm. In 
the case when $c_{0}=c_{1}$, this reduces to the challenging question of connectivity in the set of tight framelets [7].

Note that in the proof of Theorem 3.1, we have a somewhat limited control on the frame bounds of the intermediate framelets $\left\{\psi^{t}\right\}_{t \in[0,1]}$. In fact, it is possible that a lower frame bound of $\psi^{t}$ could be arbitrarily close to zero for small $t>0$. Hence, despite that the endpoints $\psi^{0}$ and $\psi^{1}$ might have nice frame bounds, the intermediate framelets constructed in the proof of Theorem 3.1 may lie outside $\mathcal{W}\left(c_{0}, c_{1}\right)$.

Example 4.1. We claim that a function $\phi \in L^{2}(\mathbb{R}), \hat{\phi}=\mathbf{1}_{[-1 / 2,1 / 2]}$ does not be belong to the $L^{2}$-norm closure of $\mathcal{W}\left(c_{0}, c_{1}\right)$ for any $0<c_{0} \leq c_{1}<\infty$. Indeed, if $\psi \in \overline{\mathcal{W}\left(c_{0}, c_{1}\right)}$, then by (1.1) and Fatou's lemma

$$
\sum_{j, k \in \mathbb{Z}}\left|\left\langle f, \psi_{j, k}\right\rangle\right|^{2} \leq c_{1}\|f\|^{2} \quad \text { for all } f \in L^{2}(\mathbb{R}) .
$$

On the other hand, a standard periodization argument shows that

$$
\sum_{j, k \in \mathbb{Z}}\left|\left\langle f, \phi_{j, k}\right\rangle\right|^{2}=\sum_{j \in \mathbb{Z}} 2^{j} \int_{-1 / 2}^{1 / 2}\left|\hat{f}\left(2^{j} \xi\right)\right|^{2} d \xi=\infty \quad \text { for all } 0 \neq f \in L^{2}(\mathbb{R}) .
$$

Therefore, it is essential that no restrictions are imposed on frame bounds of the set $\mathcal{W}$ in Theorem 2.1.

Finally, we would like to recall a related open problem posed by Larson in [12]. Is the set of all Riesz wavelets dense in $L^{2}(\mathbb{R})$ norm? Naturally, one may also ask whether the same set is pathwise connected in $L^{2}(\mathbb{R})$ norm or consider the same question for $L_{*}^{2}(\mathbb{R})$ norm. None of these questions has been answered so far.

A partial positive result in this direction has been recently obtained by Cabrelli and Molter [4], where the authors proved that any $f \in L^{2}\left(\mathbb{R}^{n}\right)$ can be approximated in $L^{2}\left(\mathbb{R}^{n}\right)$ norm by a Riesz wavelet associated to some expansive dilation matrix $A$ and some lattice of translates $\Gamma$. However, both the dilation $A$ and the lattice $\Gamma$ must vary with the accuracy of approximation. Hence, the original problem of Larson remains open, since it asks about density of Riesz wavelets for a fixed (e.g. dyadic) dilation and a fixed lattice of translates (e.g. integers). It is worth adding that Cabrelli and Molter [4] have also obtained a similar result on the density of frame wavelets as in Section 2 and independently of this work.

\section{References}

1. M. Bownik, On characterizations of multiwavelets in $L^{2}\left(\mathbb{R}^{n}\right)$, Proc. Amer. Math. Soc. 129 (2001), no. 11, 3265-3274.

2. __ Anisotropic Hardy spaces and wavelets, Mem. Amer. Math. Soc. 164 (2003), no. 781.

3. M. Bownik, E. Weber Affine Frames, GMRA's, and the Canonical Dual, Studia Math. 159 (2003), no. 3, 453-479.

4. C. Cabrelli, U. Molter, Density of the set of generators of wavelet systems, Const. Approx. (to appear).

5. X. Dai, D. Larson, Wandering vectors for unitary systems and orthogonal wavelets, Mem. Amer. Math. Soc., 134 (1998), no. 640.

6. M. Frazier, G. Garrigós, K. Wang, G. Weiss, A characterization of functions that generate wavelet and related expansion, J. Fourier Anal. Appl. 3 (1997), 883-906.

7. G. Garrigós, E. Hernández, H. Šikić, F. Soria, G. Weiss, E. Wilson, Connectivity in the set of tight frame wavelets (TFW), Glas. Mat. Ser. III 38(58) (2003), no. 1, 75-98. 
8. G. Garrigós, E. Hernández, H. Šikić, F. Soria, Further results on the connectivity of Parseval frame wavelets, Proc. Amer. Math. Soc. 134 (2006), no. 11, 3211-3221.

9. G. Garrigós, D. Speegle, Completeness in the set of wavelets, Proc. Amer. Math. Soc. 128 (2000), no. 4, 1157-1166.

10. E. Hernández, G. Weiss, A first course on wavelets, CRC Press, Boca Raton, FL, 1996.

11. M. Holschneider, Wavelets. An Analysis Tool, Clarendon Press, Oxford, 1995.

12. D. R. Larson, Von Neumann algebras and wavelets. Operator algebras and applications (Samos, 1996), 267-312, NATO Adv. Sci. Inst. Ser. C Math. Phys. Sci., 495, Kluwer Acad. Publ., Dordrecht, 1997.

13. Unitary Systems and Wavelet Sets, Proceedings of the "Fourth International Conference on Wavelet Analysis and its Applications", Macau, China, 2005 (to appear).

14. M. Paluszyński, H. Šikić, G. Weiss, S. Xiao, Generalized low pass filters and MRA frame wavelets, J. Geom. Anal. 11 (2001), no. 2, 311-342.

15. M. Paluszyński, H. Šikić, G. Weiss, S. Xiao Tight frame wavelets, their dimension functions, MRA tight frame wavelets and connectivity properties, Adv. Comput. Math. 18 (2003), $297-327$.

16. D. Speegle, The s-elementary wavelets are path-connected, Proc. Amer. Math. Soc. 127 (1999), no. 1, 223-233.

17. The Wutam Consortium, Basic properties of wavelets, J. Fourier Anal. Appl. 4 (1998), 575-594.

Department of Mathematics, University of Oregon, Eugene, OR 97403-1222

E-mail address: mbownik@uoregon.edu 